\title{
Thermally Triggered Theranostics for Pancreatic Cancer Therapy
}

Maryam Malekigorji ${ }^{1}$, Mohanad Alfahad ${ }^{1}$, Paul Kong Thoo Lin ${ }^{2}$, Stefanie Jones ${ }^{3}$, Anthony Curtis $^{1}$, Clare Hoskins ${ }^{1}$ *

1. Institute of Science and Technology in Medicine, School of Pharmacy, Faculty of Medicine and Health Sciences, Keele University, Keele, ST5 5BG, UK

2. School of Pharmacy and Life Sciences, Faculty of Health Sciences, Robert Gordon University, Aberdeen, $A B 107 Q B, U K$

3. School of Life Sciences, Faculty of Natural Sciences, Keele University, Keele, ST5 5BG, $U K$

*Corresponding author: Dr Clare Hoskins, c.hoskins@keele.ac.uk, +441782 734799 


\begin{abstract}
Hybrid iron oxide-gold nanoparticles (HNPs) are capable of drug binding onto their surface with a triggered release at elevated temperatures. The iron oxide core allows for diagnostic imaging whilst heating of the gold shell upon laser irradiation reverses drug binding. This study exploits the reversible binding of novel polyamine based drugs in order to provide specific and effective method for pancreatic cancer treatment. Here we used novel bisnaphthalamido (BNIP) based drug series. Our hybrid nanoparticles $(50 \mathrm{~nm})$ were capable of drug loading onto their surface (3:1:0.25, Drug:Fe:Au). By exploiting the surface-to-drug electrostatic interaction of a range of BNIP agents, heat triggered drug release was achieved. 12-fold reduction in $\mathrm{IC}_{50}$ after $24 \mathrm{~h}$ in vitro and 5-fold reduction of tumour retardation in vivo compared with free drug in pancreatic models after treatment with the HNP-formulation and laser irradiation. This heat activated system could provide a key platform for future therapy strategies.
\end{abstract}

Keywords: Theranostic, Pancreatic cancer, Thermo-responsive drug delivery, Laser irradiation 


\section{Introduction}

Pancreatic cancer is the $4^{\text {th }}$ most aggressive cancer in the western world [1]. Unfortunately, no major advancement in patient diagnosis, treatment or prognosis has been made in the last 40 years. Thus the number of deaths from this disease is set to rise approximately $28 \%$ by 2026 [2]. Treatment of this disease is often hindered by lack of symptoms, late diagnosis and the lag time between diagnosis, referral and treatment. Of those patients diagnosed with pancreatic cancer approximately $80 \%$ will die within a year of diagnosis and only $3 \%$ will survive 5 years [2]. In order to treat this devastating disease new technologies which overcome the major barriers to treatment must be developed. Currently the first line chemotherapy for pancreatic cancer is nucleoside analogue, gemcitabine. However, gemcitabine only proves effective in $23.8 \%$ of patients [3]. It is thought this is due to a dense stroma which surrounds the tumours and hampers drug penetration [4]. One strategy that has shown promise to increase drug penetration and increase efficiency of treatments is to couple chemotherapies onto nanoparticle carriers [5-10]. Nanoparticulates possess the ability to be either passively targeted to tumour vasculature through the enhanced permeability and retention effect [11] or actively targeted to specific sites after functionalisation with specific ligands [12]. Previous studies have shown that after coupling cytotoxics onto nanoparticles, this increased tumour penetration and resulted in more effective therapies [13-16].

Iron oxide-gold hybrid nanoparticles (HNPs) have recently been the focus of a number of investigations and are becoming increasingly applicable in biomedicine [17-20]. By using iron oxide $\left(\mathrm{Fe}_{3} \mathrm{O}_{4}\right)$ and elemental gold $(\mathrm{Au})$ within one platform, a multifunctional and stable system can be fabricated $[21,22]$. This exploits the surface chemistry and heating potential resulting from laser irradiation at the surface plasmon resonance (SPR) of the gold coating and the magnetic character of the iron oxide core, offering potential as an image guided drug carrier or theranostic agent. Reports using HNPs for delivery of anticancer agents have attached chemotherapies onto the gold surface via linker moieties or exploitation of sulfurgold chemistry [22,23].

In this study drug attachment onto the gold surface of HNPs via electrostatic interaction is explored for the first time as a potential loading and release strategy. Electrostatic interactions with polymers or stabilising agents are commonly used for functionalisation of nanoparticles but, to the best of our knowledge, have not been reported as a mechanism for stimuli responsive drug delivery from HNPs. Stimuli responsive drug delivery occurs when drug 
release is initiated by a change in environmental conditions, such as temperature or $\mathrm{pH}$. The rationale is that positively charged drug molecules will form electrostatic interactions with the overall negatively charged gold surface of the HNPs. Electrostatic bonds are chargecharge interactions which may be cleaved at increased temperatures when the energy threshold required to break these bonds is reached [24]. Here we exploit the nano-heating properties of these HNPs in order to break the electrostatic binding of drug molecules which results in localised triggered drug release (Fig. 1A). This reversible binding mechanism allows for controllable release of pharmaceutical agents in their parent state unhindered by addition of linkers or permanent bonds, hence no reduction in drug activity is experienced as is so commonly observed in controlled release systems [25].

Here a series of novel bisnaphthalimide drug analogues with a chain of 20 atoms separating two naphthalimido residues will be used (Fig. 1B). Brana et al. reported bisnaphthalimides as potent chemotherapeutic agents [26-29]. Bisnaphthalimide derivatives are identified to possess significant anti-tumour activity in both murine and human cancerous cells [26-29]. Due to the presence of planar aromatic moieties, these molecules can intercalate within the DNA by entry via the major groove [30]. Hoskins et al. showed that the potency of an insoluble bisnaphthalimido compound could be enhanced in vitro and in vivo in pancreatic models using a nano-aggregate of poly(allylamine) modified with cholesteryl chains. This aggregate increased drug solubilisation and resulted in more rapid drug uptake into pancreatic cancer BxPC-3 cells compared with the free drug. A similar clinical effect was experienced with the clinically used drug gemcitabine at 8 -fold less dose [31].

In this study, a number of soluble bisnaphthalamide analogues with a backbone containing different number of charged amino residues were electrostatically conjugated onto the HNP surface (Fig. 1A). In order to determine the effect of drug charge on the loading concentration and subsequent drug release, we used bisnaphthalimido: 1,20 diaminoicosane (BNIPDi) (containing no amino residues), 1,12 diaminooctane dihydrogen bromide (BNIPd), propyl spermine tetrahydropbromide (BNIPSpm), and propyl 3,3'-butane-1,4diylbis(sulfanediyl)bis(propan-1-amine) (BNIPds) (Fig. 1B). The temperature dependant release and biological activity of the novel systems was evaluated both in vitro and in vivo in order to ascertain clinical relevance of these systems for pancreatic cancer therapy. 


\section{Experimental}

All chemicals and solvents used were commercially available and purchased from Sigma Aldrich (UK) unless otherwise stated. Anhydrous solvents were purchased as such or dried using standard techniques. Cell culture media was purchased from Thermofisher (UK). All cell lines used were purchased from ATCC, LGC Standards UK and mice were purchased from Charles River, UK.

\subsection{Synthesis and characterisation of drug molecules}

The drug compounds were synthesised according to the methodology outlines in the Supplementary Information (SI-Methods 1.0). The drugs were characterised using ${ }^{1} \mathrm{H},{ }^{13} \mathrm{C}$ and ${ }^{19} \mathrm{~F}$ NMR spectra which were recorded at $300 \mathrm{MHz}$ using a Bruker Spectrospin DPX 300 Spectrometer and at $400 \mathrm{MHz}$ using a Bruker Ascend 400 spectrometers; Multiplicities were recorded as broad peaks (br), singlets (s), doublets (d), triplets $(\mathrm{t})$, quartets $(\mathrm{q})$, quintets (qu), double doublets (dd), and multiplets (m). All NMR samples were made up in deuterated solvents with all values quoted in ppm relative to tetramethylsilane (TMS) as an internal reference. Coupling constants ( $J$ values) are reported in Hertz $(\mathrm{Hz})$. High resolution mass spectrometry analyses were performed using a ThermoFisher Scientific LTQ Orbitrap XL hybrid ion trap-orbitrap mass spectrometer. Samples were introduced to the spectrometer as solutions in dichloromethane/methanol with ammonium acetate added. All compounds analysed gave satisfactory data at high resolution as compared to predicted ionisation patterns.

\subsection{HNP - drug conjugation and characterisation}

HNP-Drug conjugation: To a solution of HNPs $\left(1 \mathrm{mgmL}^{-1}, 5 \mathrm{~mL}\right)$ a $5 \mathrm{mg}, 12.5 \mathrm{mg}$ or $25 \mathrm{mg}$ of drug was added along with $25 \mathrm{mg}$ of poly(ethylene glycol) thiol. The solution was stirred for $3 \mathrm{~h}$ at $25{ }^{\circ} \mathrm{C}$. The particles were magnetically separated and extensively washed with deionised water.

Drug attachment was quantified by reverse phase high performance liquid chromatography (HPLC) (Prominence, DEGASSER, LC20AD, SHIMADZU) using a fluorescent detector at excitation $234 \mathrm{~nm}$ and emission 394 wavelengths (Jasco, PU-980, Japan; column C18(2), $150 \times 4.60 \mathrm{~mm} 5$ micron, flow rate $1 \mathrm{~mL} / \mathrm{min}$, injection volume $20 \mu \mathrm{L}$ ). A buffer was prepared by adding $0.432 \mathrm{~g}$ octane sulfonic acid and $1.64 \mathrm{~g}$ sodium acetate to $200 \mathrm{~mL}$ of deionised water. Then the $\mathrm{pH}$ of the solution was reduced to $\mathrm{pH} 4.5$ by the addition of hydrochloric 
acid. Mobile phase was prepared by mixing buffer and acetonitrile with the percentage of $70 \%$ and $30 \%$, respectively. A flow rate of $1 \mathrm{~mL} \mathrm{~min}^{-1}$ was employed. A calibration was run using drug solutions dissolved in 50:50 $\mathrm{H}_{2} \mathrm{O}$ : Aceotnitrile with the concentration of 100-1.56 $\mu \mathrm{gmL}^{-1}\left(\mathrm{R}^{2}=0.9999\right)$. All measurements were run in triplicate and recorded as average values.

Formulations with the highest drug loading concentrations $(5 \mathrm{~mL})$ were freeze dried using a Heto PowerDry LL3000, Thermo-Fisher UK. The freeze dried samples were analysed by FTIR spectroscopy using a diamond tipped attenuated total reflectance attachment (Nicolette iS5 with iD5 ATR, Thermo-Fisher UK) at resolution of $4 \mathrm{~cm}^{-1}$ and 64 scans were run at room temperature with background subtraction in order to qualitatively verify drug presence.

Aqueous formulations were characterised at room temperature on the Luminescence Spectrometer (Varian, Cary Eclipse Fluorescence Spectrophotometer, USA) with excitation wavelength set at $234 \mathrm{~nm}$. The samples were scanned for emission between $200-600 \mathrm{~nm}$ at $400 \mathrm{nmsec}^{-1}$, and the spectra were collected and analysed by the provided software. This software controls all aspects of the system, such as control of the slits at the entrance and exit ports of the spectrograph, detector gain, blank-subtraction, system correction files and automated batch-processing.

Zeta potential measurements were carried out using a photon correlation spectrometer (PCS, Zetasizer Nano-ZS, Malvern Instruments, UK). Samples were diluted in deionised water to make $1 \mathrm{mgmL}^{-1}$ of HNPs and sonicated for $30 \mathrm{~s}$ before measuring the surface charge of the particles at $25^{\circ} \mathrm{C}$.

Photon correlation spectroscopy (PCS) carried out in order to evaluate particle size before and after drug conjugation. Samples were analysed at $25^{\circ} \mathrm{C}$ using a PCS, Zetasizer Nano-ZS, Malvern Instruments, UK). Samples were run in triplicate and an average value recorded. Additional size measurements were carried out using transmission electron microscopy (TEM). TEM imaging was carried out using the JEOL JEM-1230 (JEOL, Japan) transmission electron microscope. Prior to imaging, formvar coated copper grids were prepared. The samples were pipetted $(10 \mu \mathrm{L})$ onto the grids and allowed to air dry before imaging. Sample diameter was measured and recorded for 20 particles and an average size recorded. 


\subsection{Stability of HNP-drug formulations}

The formulations were tested as both aqueous solutions and dried formulations. These studies was run at both room temperature and $4{ }^{\circ} \mathrm{C}$ over the period of 4 weeks. For the aqueous formulations, $1 \mathrm{~mL}$ of each formulation (drug concentration $=0.5 \mathrm{mgmL}^{-1}$ ) was kept at room temperature and $4{ }^{\circ} \mathrm{C}$ and the supernatant was analysed each week with HPLC. For studying the stability of powder formulations $1 \mathrm{~mL}$ of each formulation with the same mentioned concentration was freeze dried. Each week samples were reconstituted in deionised water and the concentration of released drug investigated via HPLC as previously described. All measurements were run in triplicate and recorded as average values.

\subsection{In vitro drug release}

Formulations ( $\left.2 \mathrm{~mL}, 500 \mu \mathrm{gmL}^{-1}\right)$ were placed into visking tubing (12-14 KDa) and dialysed against $200 \mathrm{~mL}$ deionised water / cell culture media (RPMI, pH adjusted to 7.4, 4.6 \& 3.6) at $\left(20{ }^{\circ} \mathrm{C}, 30{ }^{\circ} \mathrm{C}, 40{ }^{\circ} \mathrm{C}, 44{ }^{\circ} \mathrm{C}, 50{ }^{\circ} \mathrm{C} \& 60{ }^{\circ} \mathrm{C}\right)$. At selected time points $(0.083 \mathrm{~h}, 0.17 \mathrm{~h}, 0.33 \mathrm{~h}$, 0.5 h, 1 h, 4 h, 6 h, 8 h, 24 h, 48 h, 72 h, 144, 168 and 216 h) a sample of the exterior solution $(1 \mathrm{~mL})$ was removed and replaced with $1 \mathrm{~mL}$ deionised water at the specified time points. All samples were diluted with $1 \mathrm{~mL}$ of acetonitrile and drug concentrations were analysed via HPLC as previously described. The experiment was carried out in triplicate and the peak area compared to a calibration of the free drug dissolved in acetonitrile/ $\mathrm{H}_{2} \mathrm{O}(50: 50)\left(\mathrm{R}^{2}=0.999\right)$.

\subsection{In vitro evaluation}

Human pancreatic adenocarcinoma (BxPC-3), human epithelial ductal carcinoma (PANC-1) and human myeloid monocyte (U937) cells were cultured in RPMI (BxPC-3 \& U937) AND DMEM (Panc-1) media. The media was supplemented with $1 \%$ penicillin/streptomycin and $10 \%$ foetal bovine serum ( $1 \%$ of L-glutamine was also added to PANC-1 \& U937 media). U937 cells were differentiated with $0.02 \%$ of phorbol-12-myristate 13-acetate (PMA) (50 $\mu \mathrm{gmL}^{-1}$ in PBS) was added to the cells suspension in order to transform them into macrophage like cells before testing.

\subsubsection{MTT Assay}

Cells $(100 \mu \mathrm{L}, 15000$ cells/well) in exponential growth phase were seeded into 96 well flat bottomed plates and incubated for $24 \mathrm{~h}$ at $37{ }^{\circ} \mathrm{C}$ with $5 \% \mathrm{CO}_{2}$. After $24 \mathrm{~h}$, the media was replaced with various concentrations of gemcitabine, free bisnaphthalamide based drugs, naked HNPs and novel formulations, diluted in cellular growth medium $\left(0.01 \mu \mathrm{gmL}^{-1}-500\right.$ 
$\left.\mu \mathrm{gmL}^{-1}\right)$. After $24 \mathrm{~h}$, the drug solutions were removed and washed with fresh media. 3-[4, 5dimethylthiazole-2-yl]-2, 5-diphenyl tetrazolium (MTT, $50 \mu \mathrm{L}, 5 \mathrm{mgmL}^{-1}$ in PBS) was added to the wells and plate was incubated $\left(37^{\circ} \mathrm{C}\right.$ with $\left.5 \% \mathrm{CO}_{2}\right)$ for $4 \mathrm{~h}$. After this time, the MTT solution was removed from the wells. The remaining purple formazan complexes were dissolved in DMSO $(100 \mu \mathrm{L})$ and the absorbance of the plates was read at $570 \mathrm{~nm}$ using a microplate reader (Tecan, infinite 200 pro, GmbH 5082, Australia). Percentage cell viability and $\mathrm{IC}_{50}$ was calculated relative to positive and negative controls.

\subsubsection{Trypan blue cytotoxicity test}

Cells (1 mL, 50000 cells/well) were seeded into 12-well flat bottomed plates and incubated for $24 \mathrm{~h}$ at $37{ }^{\circ} \mathrm{C}$ with $5 \% \mathrm{CO}_{2}$. The media was replaced with drugs, HNPs and novel formulations at various concentrations $\left(0.01 \mu \mathrm{gmL}^{-1}-500 \mu \mathrm{gmL}^{-1}\right)$. Cells were incubated for $24 \mathrm{~h}$, subsequently the media was removed and cells were washed 3 times with PBS. The cells were trypsinised and re-suspended in fresh media. A mixture of $50 \mu \mathrm{L}$ of cells and 50 $\mu \mathrm{L}$ of trypan blue solution was placed in an automated cell counter (Invitrogen Countess ${ }^{\circledR}$, UK) and viable cells were counted. Percentage cell viability and $\mathrm{IC}_{50}$ were calculated in relation to control cells.

\subsubsection{Cellular uptake of formulations}

Cells (3 mL, 150000 cells/ well) were seeded into 6-well plates and incubated for $24 \mathrm{~h}$ at $37^{\circ} \mathrm{C}$ with $5 \% \mathrm{CO}_{2}$. The media was replaced with $50 \mu \mathrm{gmL}^{-1}$ and $100 \mu \mathrm{gmL}^{-1}$ of different bisnaphthalamide based drugs, their hybrid formulations and gemcitabine and incubated for 1 $\mathrm{h}$ and $4 \mathrm{~h}$. The medium was removed and each well was washed with $1 \mathrm{~mL}$ PBS before the addition of $185 \mu \mathrm{L}$ trypsin into each well. Cells re-suspended in $1 \mathrm{~mL}$ media and viable cells were counted using an automated cell counter (Invitrogen Countess ${ }^{\circledR}$, UK). Cells $(100,000)$ were transferred into eppendorf tubes and centrifuged ( $800 \mathrm{rpm}, 5 \mathrm{~min})$. The supernatant was removed and cells were resuspended in DMSO:water (1:1) (for samples containing bisnaphthalamide derivatives) or water (for samples containing gemcitabine). The drug concentration was quantified using reverse phase HPLC. Analysis of the bisnaphthalimides was carried out via HPLC as described in the drug loading methodology. Gemcitabine quantification was carried out using an HPLC instrument with UV detector (Perkin Elmer, Flexar Autosampler, column: SPHERISORB ODS $25 \mu \mathrm{m}$, length $250 \mathrm{~mm}$, internal diameter $4.6 \mathrm{~mm})$ and a mobile phase of water:acetonitrile (30:70). The samples were detected at 234 
$\mathrm{nm}$ with flow rate of $1 \mathrm{~mL}$.

\subsubsection{Thermo-responsive cytotoxicity}

Cells (3 mL, 150000 cells/well) in their exponential growth phase were seeded into 6-well plates and incubated for $24 \mathrm{~h}$ at $37{ }^{\circ} \mathrm{C}$ with $5 \% \mathrm{CO} 2$. Cells were treated with $50 \mu \mathrm{gmL}^{-1}$ of drugs and formulations and incubated for $1 \mathrm{~h}$. Then, cells were further incubated at $25^{\circ} \mathrm{C}, 44$ $\mathrm{VC}$ and $60{ }^{\circ} \mathrm{C}$ for $0.5 \mathrm{~h}$, in order to investigate the effect of temperature on cell cytotoxicity. Then the drug solutions were removed and cells were washed with fresh media to remove any excess drug. Fresh media was then added to each well. After $24 \mathrm{~h}$ incubation $\left(37{ }^{\circ} \mathrm{C}, 5 \%\right.$ $\mathrm{CO}_{2}$ ), media was removed and cells were washed with PBS. The cells were counted using trypan blue exclusion as described above.

\subsubsection{AFM topography imaging}

Cells ( $3 \mathrm{~mL}, 150000$ cells/well) were seeded in 6-well plates containing glass coverslips and incubated for $24 \mathrm{~h}$ at $37{ }^{\circ} \mathrm{C}$ and $5 \% \mathrm{CO}_{2}$. After $24 \mathrm{~h}$ the media was replaced with gemcitabine, BNIPSpm, HNP-BNIPSpm and naked HNPs solution $\left(50 \mu \mathrm{gmL}^{-1}\right)$ and further incubated for $1 \mathrm{~h}$ and $4 \mathrm{~h}$. After abundant washing with PBS, cells were fixed with $1 \mathrm{~mL}$ gluteraldehyde $(2.5 \%$ in PBS) for 10 min. Fixed cells were washed 5 times with PBS and mounted on glass slides. Cell topography was imaged with a Bruker Catalyst Atomic Force Microscope (Bruker, Germany) using ScanAsyst mode in air (using silicon tip (Bruker, T: $650 \mathrm{~nm}, \mathrm{~L}: 115 \mu \mathrm{m}, \mathrm{W}: 25 \mu \mathrm{m})$ on nitride lever).

\subsection{In vivo evaluation}

Female $\mathrm{Nu} / \mathrm{Nu}$ mice, 5 weeks of age (Charles River, UK) were kept in pathogen-free conditions (weight of mice was 20-25 g). All procedures and animal care were carried out according to Project License PPL 70/8806 granted by the UK Home Office. Human pancreatic cancer cell line BxPC-3 was cultured to $90 \%$ confluence in RPMI 1640 supplemented with $10 \%$ fetal bovine serum and $1 \%$ penicillin streptomycin. The cells were washed twice with cold PBS and harvested with trypsin for $10 \mathrm{~min}$ at $37^{\circ} \mathrm{C}$. The cells were washed three times with PBS and resuspended in 50:50 media:PBS. The tumour cell suspension $\left(3.0 \times 10^{6}\right.$ cells in $\left.100 \mu \mathrm{L}\right)$ was injected subcutaneously (s.c.) in the right flank of each mouse. When the tumour became palpable (approximately after one week), measurements in two dimensions with vernier calipers were carried out twice a week and 
volume tumours calculated according to equation (1).

$$
\mathrm{V}=4 / 3 \pi[(\mathrm{D} 1+\mathrm{D} 2) / 4]^{3}
$$

Once tumours had reached $0.1 \mathrm{~cm}^{3}$ therapeutic studies commenced. The mice were grouped into 8 arms $(\mathrm{n}=5)$ of control, HNP, BNIPSpm, HNP-BNIPSpm all with and without laser irradiation. Drug administration was $3 \mathrm{mgKg}^{-1}$ with equivalent HNP concentration used in the HNP control. Doses were injected intratumorally (I.T.) using a 26 gauge needle (Vet-Tech, $\mathrm{UK}$ ) at a maximum of $100 \mu \mathrm{L}$. The dose was administered one dose per week over a four week period. Where laser irradiation was required this was carried out $24 \mathrm{~h}$ after dosing under anaesthetic. The tumour was irradiated at $1064 \mathrm{~nm}$ for $20 \mathrm{sec}$ using a ML-LASER-YB5 Qswitched Nd:YAG Laser Treatment System (WeiFang MingLiang Electronics Company Ltd., China). Pulse width: $10 \mathrm{~ns}$, pulse repetition frequency: $6 \mathrm{~Hz}$, laser spot diameter: $3 \mathrm{~mm}$, cooling system: water cooled with airflow cooling. The beam was collimated through concave lenses to a $1 \mathrm{~mm}$ diameter. Any mouse whose tumour volume reached $0.9 \mathrm{~cm}^{3}$ was sacrificed in line with good practice guidelines [32].

\subsection{Statistical analysis}

A simple two tailed t-test was carried out in excel to determine the statistical significance of results, whereby $\mathrm{p} \leq 0.01$ was significant.

\section{Results and Discussion}

\subsection{Synthesis and characterisation of drug molecules}

The drug molecules used in this study were synthesized using established methodology according to the general scheme shown in Fig. 2 [33]. The identity of these compounds was confirmed by NMR spectroscopy and Mass spectrometry (SI-Fig. 1).

\subsection{HNP-Drug conjugation and characterisation}

The HNP preparation and characterisation has been reported previously [21,22] along with their ability to undergo triggered heating upon laser irradiation in agar phantoms [17,34], in vitro and in situ in tumour bearing mouse cadavers [35,36]. Drug attachment was carried out at three drug:HNP weight ratios (5:1, 2.5:1 and 1:1 based on Fe weight of HNP) for all four bisnaphthalamide based drugs. Higher initial drug weight concentrations were not investigated due to the lack of aqueous solubility of the drug analogues over $5 \mathrm{mgmL}^{-1}$. The data suggested that BNIPDi could not be conjugated onto the surface of HNPs due to the lack 
of protonated amino group (Fig. 3A), which is essential for electrostatic interaction, or any other driving force for conjugation. FTIR spectral analysis (SI-Fig.2A), fluorescence spectroscopy (SI-Fig.3A) and the lack of shift in zeta potential measurement of the BNIPDi formulations (Fig. 3B) confirmed that no drug attachment had occurred. As a result, it was decided not to study these formulations further. Photon correlation spectroscopy (SI-Table 1) was inconclusive in terms of nanoparticle size. This was due to the fact that aggregation was occurring on measurement, particularly in the HNP with no drug coating. Indeed the measurements appeared to become more realistic after drug conjugation, this is likely to be due to the stabilizing effect of the drug on the HNPs in solution, hence reducing their tendency to aggregate in solution. Size measured via TEM showed that there was no notable increase in particle size after drug conjugation onto the surface of the HNPs.

Drugs with amino groups within their backbone did successful achieve binding onto the gold surface (Fig. 3A) in agreement with Aslam et al., who reported that gold has a strong affinity towards amino groups [37]. The successful binding between BNIPd and the HNP is due to electrostatic interaction between the two positively charged amino groups within this drug and the gold coating, as observed by the appearance of the $\mathrm{N}-\mathrm{H}\left(700-800 \mathrm{~cm}^{-1}\right), \mathrm{C}=\mathrm{O}(1658$ $\left.\mathrm{cm}^{-1}\right)$ and $\mathrm{CH}_{2}\left(2915 \mathrm{~cm}^{-1}\right)$ stretching vibrations in the FTIR spectrum (SI-Fig.2B). Additionally, the zeta potential measurement showed a shift from $-4 \mathrm{mV}$ to $+26 \mathrm{mV}$ indicating the presence of amines. The presence of fluorescent emission at $424 \mathrm{~nm}$ further substantiated this (SI-Fig.3B). The loading pattern of BNIPd onto the HNP did not follow a linear relationship. In fact, after 2.5:1 drug:HNP loading the graph plateaued (Fig. 3A) suggesting weak electrostatic interactions being formed and broken due to competition of drug molecules for space on the HNP surface with surface saturation not being achieved.

For BNIPSpm, increasing drug loading concentration resulted in greater drug attachment onto the nanoparticle surface (Fig. 3A). This could be due to the number of protonated amino groups present in the drug backbone (four in the case of BNIPSpm when compared to two in BNIPd), resulting in stronger electrostatic interactions between the drug and HNP. The absence of a plateau in the graph suggests that further conjugation could be possible and the HNP surface was not saturated; however, the physicochemical properties of the drug in solution hindered further attachment. BNIPSpm loaded onto the HNPs exhibited a linear correlation between the drug concentration solution fed to the particles and the attached drug concentration. For example our data showed that $5 \mathrm{mgmL}^{-1}$ of loading BNIPSpm solution 
conjugated to the surface of HNPs at higher concentration compared to the other drugs investigated in this study. Approximately $3.4 \mathrm{mgmL}^{-1}$ of BNIPSpm from the initial $5 \mathrm{mgmL}^{-1}$ loading concentrations was attached to the HNP, hence achieving a binding efficiency of $68 \%$. A shift in zeta potential was observed (Fig. 3B) which correlated well with the fluorescence and FTIR data (SI-Fig.2C \& 3C), confirming the presence of drug on the HNP surface.

BNIPds is conjugated to the HNP by dative covalent bonding of the sulfide residues with the $\mathrm{Au}$ surface. $\mathrm{Au}-\mathrm{S}$ bonds are relatively strong, approximately $40 \mathrm{kcal} \mathrm{mol}^{-1}$ [38], compared with weaker Van der Waals forces. For BNIPds the graph did plateau at higher drug ratios (Fig. 3A) suggesting the particle surface was saturated and no more attachment was possible. Given the nature of the strong sulfide interaction achieved with BNIPds in addition to the electrostatic binding opportunities arising from the two amino groups, when compared with the potential for only electrostatic interactions of the other drugs investigated it is postulated that a more rigid binding occurs for BNIPds with less molecular flexibility, utilising greater surface area and hence leading to lower binding saturation concentrations. The reduced drug conjugation at the HNP surface was confirmed by a shift in the zeta potential measurement from $-4 \mathrm{mV}$ to $+10 \mathrm{mV}$. The presence of electronegative sulfur atoms in BNIPds may have contributed to the reduced zeta potential shift when compared to BNIPSpm and BNIPdi.

Previous studies have shown that doxorubicin can be absorbed onto colloidal gold surfaces and electrostatically attached. In the case of this drugs, it has been shown that the hydrophobic moieties within the structure drive the molecule towards the gold surface and secondly the charge within the compound resulting from the one primary amine allow for electrostatic attachment [39]. The mechanism of bisnaphtalamide drug attachment onto the HNP surface may undergo a similar mechanistic approach to attachment, however, the multiple amine charges resulting from the polyamine chain far outweigh the hydrophobicity of the naphthalamide moieties within the drug structure resulting in a freely soluble drug compound, unlike in the case of doxorubicin. Hence, the mechanism in this case may solely rely on charge-charge interaction, without the initial push from the hydrophobic aromatic groups. More work is required in order to confirm the exact mechanism of the electrostatic interactions obtained for these drugs. 


\subsection{Stability of HNP-drug formulations}

Stability studies of the drug-HNP formulations (Fig. 3C\&D), including reconstituted freezedried samples, were carried out in aqueous solution at $4{ }^{\circ} \mathrm{C}$ and room temperature $\left(25^{\circ} \mathrm{C}\right)$. These results showed that BNIPds did not dissociate from the HNP surface, which was expected due to the covalent linkage between sulfide and the Au surface of the HNP. BNIPd became almost totally unattached from the HNPs at both $4{ }^{\circ} \mathrm{C}$ and $20{ }^{\circ} \mathrm{C}$ probably due to the weak electrostatic interaction being broken down. BNIPSpm however, remained in formulation with more than $80 \%$ of drug remaining attached to the HNPs in solution over the duration of the study. This is probably due to the increased number of charges in the drug resulting in a greater electrostatic force and tighter binding. The freeze-dried formulations were optimal with increased longevity observed.

\subsection{In vitro drug release}

Drug release was carried out under 'sink' conditions in aqueous solution (Fig. 4A) and in cell culture media with $\mathrm{pH}$ adjusted to mimic in vivo conditions (Fig. 4B). Studies with BNIPd showed that $100 \%$ of the drug was released rapidly at $20{ }^{\circ} \mathrm{C}$ (almost $80 \%$ after $0.5 \mathrm{~h}$ ) (SIFig.4); it is proposed that the charge-charge interaction is not strong enough for long-term stability of the formulation. Such instability and rapid dissociation from the formulation renders it unsuitable as a delivery system for this drug. In general, the BNIPSpm release from formulations in both water and culture media showed biphasic patterns, which include a sharp release in the first $10 \mathrm{~h}$ followed by a gradual release for the remainder of the experiment. Less than $10 \%$ of drug had been released at a biologically relevant temperature i.e. $37{ }^{\circ} \mathrm{C}$. At $60{ }^{\circ} \mathrm{C}$ higher drug release occurred, after only $0.5 \mathrm{~h}$ almost $40 \%$ of drug had been released from the formulations, which may also be achievable upon irradiation of the HNPs at the SPR over short time durations without detriment to surrounding cells. The release profiles of BNIPSpm formulations indicate that they are superior to the BNIPd formulations. It is hypothesized that the increased number of charged residues in BNIPSpm results in stronger electrostatic interactions which requires a larger input of energy to dissociate it from the HNP surface, thus the formulation is more stable at lower temperatures and only releases drug after heat stimulation.

In our study BNIPSpm drug release in culture media at biologically relevant temperatures $\left(37^{\circ} \mathrm{C}\right)$ and $\mathrm{pH}(7.4)$ followed the same pattern of drug release seen in water, whereby increasing the temperature (from $37{ }^{\circ} \mathrm{C}$ to $44{ }^{\circ} \mathrm{C}$ ) enhanced the drug 
release rate. A decrease in $\mathrm{pH}$ to intracellular levels resulted in further drug release. At $\mathrm{pH}$ 3.6, there was a significant increase in drug released from BNIPSpm-HNP compared to physiological $\mathrm{pH}$. Thus, BNIPSpm-HNP formulations exhibited $\mathrm{pH}$ and thermo-responsive drug delivery properties. Therefore, it is envisaged that after accumulation of the HNPs inside the endosomes or lysosomes together with the low environmental $\mathrm{pH}$ accompanied by heating through laser irradiation will result in rapid drug release from the nanoparticles. Thus, allowing free drug to enter the nucleus to interact with DNA for therapeutic effect. As expected, due to the robust binding interaction of BNIPds, less than $2 \%$ of the drug was released after $72 \mathrm{~h}$ at $60{ }^{\circ} \mathrm{C}$ (SIFig.4). Therefore, BNIPds formulations cannot be utilised for thermally triggered drug delivery.

Based on the above results, only BNIPSpm formulation studies were carried forward for in vitro and in vivo testing on pancreatic cancer cells.

\subsection{In vitro evaluation}

Cell viability of BxPC-3, Panc-1 and U937 cells exposed to HNP-BNIPSpm was determined using both the MTT assay and trypan blue exclusion (Fig. 5A). BxPC-3 and Panc-1 cell lines were chosen in this study in order to gain realistic information on the potential of the formulations in pancreatic therapy. Cancer is not defined as one cell type, indeed it is a cellular mutation which leads to rapid proliferation, this can occur in multiple cell types within one cancer, which may react differently to treatment. Therefore, looking at more than one cell line may give more meaningful information and correlation between cellular response and in vivo outcome. In this study, generally, both cytotoxicity assays exhibited comparable results in the pancreatic cell lines. The naked HNPs did not present a remarkable dose response effect on cell viability up to $50 \mu \mathrm{gmL}^{-1}$ ( $\mathrm{p}>0.05$ ) (SI-Fig.5). However, after $24 \mathrm{~h}$ incubation with the highest concentration of HNPs $\left(100 \mu \mathrm{gmL}^{-1}\right)$ a $19-23 \%$ decrease in viability was obtained $(\mathrm{p}<0.05)$. As with any drug delivery vehicle, inherent toxicity is not desirable. In this case it is not a concern since the concentrations tested are more potent than would be expected to be administered in the drug formulation to a patient in the clinic. Over the concentration ranges and time points tested there was no $\mathrm{IC}_{50}$ evident for either the HNP (SI-Fig.4A) or gemcitabine (SI-Fig.4B). As gemcitabine is the gold standard for clinical treatment it was interesting to note that for BNIPSpm, after only $24 \mathrm{~h}$ an $\mathrm{IC}_{50}$ 
value was detected, hence this indicates that the novel drug is more rapid and potentially more toxic than the clinical gold standard.

Consistently in the pancreatic cancer cell lines (BxPC-3 and Panc-1), when BNIPSpm was conjugated onto the HNP a decrease in $\mathrm{IC}_{50}$ was achieved. This was particularly evident in the case of $\mathrm{BxPC}-3$ cells where a significant $(\mathrm{p}<0.01)$ reduction in $\mathrm{IC}_{50}$ values was observed in both the MTT and trypan blue exclusion assays with an $\mathrm{IC}_{50}$ as low as $1.37 \mu \mathrm{gmL}^{-1}$ in BxPC-3 cells (Fig. 5A). The increase in activity may be due to the internalisation mechanism of the drug. It is hypothesized that the free drug is internalised into the cell via a diffusion driven pathway which is time consuming. When studying the data of gemcitabine cellular uptake (SI-Fig.5C) the amount of drug internalised per cell is greater than that of BNIPSpm. However, even with this increased in internalisation, no toxicity was observed. It is widely reported that the majority of nanoparticulate suspensions and colloids are internalised via endocytosis [40]. This mechanism of 'cellular drinking' is more rapid and the process of engulfing and membrane rearrangement results in greater cellular uptake of molecules compared to the diffusion mediated routes. Hence, greater internalisation is the likely reason for the decreased $\mathrm{IC}_{50}$ of HNP-BNIPSpm (Fig. 5A). The cellular uptake data shown in Fig. 5B, confirms this theory, where an increase as high as 8-fold of BNIPSpm in BxPC-3 cells was observed after HNP conjugation and $4 \mathrm{~h}$ incubation.

In order to gauge the effect of the novel formulation on the immune system, U937 human monocyte cells were differentiated in order to mimic macrophage like activity. Interestingly, greater cellular uptake was observed with the free BNIPSpm compared to the HNP bound drug (Fig. 5B). This result is quite surprising as monocyte cells typically 'eat' and 'destroy' any foreign bodies in the bloodstream. Perhaps the presence of the poly(ethylene glycol) mimics biological molecules and avoid being endocytosed by the cells. More likely however, is that fact that these are a noncancerous derived cell line and particularly after differentiation they are not proliferating rapidly and hence endocytosis may take longer. Hence, for U937 cells, drugs enter cells preferentially via the diffusion mediated pathways. This is reflected in the cytotoxicity data presented in Fig.5A where a significant increase in $\mathrm{IC}_{50}$ was observed with HNP-BNIPSpm conjugation. 
In order to determine whether reversal of drug binding resulted in toxicity enhancement in vitro, trypan blue cell viability counting was carried out after $24 \mathrm{~h}$ exposure to $50 \mathrm{\mu gmL}^{-1}$ of the formulation. The protocol carried out was the same except these cells had been incubated at increased temperatures $\left(44{ }^{\circ} \mathrm{C}, 60{ }^{\circ} \mathrm{C}\right)$ for 0.5 h. This exposure duration was deemed sufficient to initiate drug release (Fig. 4) without causing any adverse effect to the cells. Indeed, at increased temperatures the $\mathrm{IC}_{50}$ values dropped although this was only found to be statistically significant $(\mathrm{p}<0.01)$ in the case of BxPC-3 cells exposed to $60{ }^{\circ} \mathrm{C}$ (Fig. 5C). Although, this was only a crude experiment and it is not certain if it properly reflects the in vivo behavior of these drugs after heat initiated release.

Further investigation into the cellular state was studied after drug/formulation exposure using AFM imaging technique. The latter has often been used as an early indicator of in vitro fate since detailed cellular morphology can be observed. Figure 5D-E shows the topography images for all three cell lines undergoing the various treatment after $1 \mathrm{~h}$ exposure. Strikingly, the cancer cell lines incubated with HNPBNIPSpm (Fig.5D5, E5) showed completely different profiles to controls (Fig. 5D1, E1) with total cellular rearrangement and breakdown starting to occur even after this short time exposure. In common with the cytotoxicity (Fig. 5A) and uptake data (Fig. 4B), those cells treated with free drug (Fig.5D4, E4) also appeared unhealthy, but to a lesser extent. Unsurprisingly, the HNP (Fig.5D2, E2) or the gemcitabine treated cells (Fig.5D3, E3) did not look notably different to the control cells (Fig. 5D1, E1), with smooth, well defined and communicative surface. The U937 cells (Fig.5F) followed a similar trend to their cytotoxicity and uptake data, whereby the cells treated with free drug (Fig.5 F4) looked less rounded and smooth compared to the control cells (Fig. 5F1). In agreement with the cytotoxicity data, those cells treated with HNP alone (Fig.5 F2) or gemcitabine (Fig.5 F3) did not look notably different from the control cells.

\subsection{In vivo evaluation}

The novel formulation (HNP-BNIPSpm) was tested on subcutaneous BxPC-3 xenograft models on the back flank of $\mathrm{Nu} / \mathrm{Nu}$ mice to determine its in vivo potential as an anticancer agent. Although in vitro studies do give some indication of the in vivo fate, often large deviation in response between cell lines and in vivo studies is 
observed. In our study, mice with tumours (approx. $0.1 \mathrm{~cm}^{3}$ ) were dosed at $3 \mathrm{mgKg}^{-1}$ once per week over a 4-week period. In order to exploit the thermo-responsive properties of these formulations, laser irradiation was used. Extensive previous studies have been carried out in order to optimize the laser treatment duration in order to achieve temperatures that are likely to initiate drug release without adverse effects to tissue $[34,35]$.

Figure 6 A 1 shows a tumour excised from a control group mouse. Unfortunately, the mice from this group and the HNP alone group, did not complete the duration of the study. This is because their tumour volumes reached the maximum humane limit $(0.9$ $\mathrm{cm}^{3}$ ) and hence the study had to be halted early. Looking at the tumour images (Fig.6 A) in conjunction with weight (Fig.6 B) and volume (Fig.6 C) data it is evident that no reduction in tumour size was observed (Fig.6 A 3 \& 4) for the HNP treatment and control group. However, those mice treated with BNIPSpm resulted in a significant tumour retardation (Fig.6 A 5 \& 6). Looking across the control, HNP and BNIPSpm groups, no significant $(\mathrm{p}>0.01)$ reduction in tumour weight or volume was observed due to laser irradiation (Fig.6 2, 4 \& 6). Mice treated with HNP-BNIPSpm with no laser irradiation (Fig.6 A7) experienced tumour retardation to a slightly lesser extent than for those treated with the free drug (Fig.6 A 5), however, this was not deemed to be significant $(\mathrm{p}=0.234)$. Most excitingly, the HNP-BNIPSpm treated mice which underwent laser irradiation experienced a significant enhancement $(p<0.01)$ in tumour retardation (Fig.6 A8) compared to the free drug groups. Here, 50\% reduction in tumour weight (Fig.6B) and volume (Fig. 6C) were experienced. It is estimated that the control and HNP group tumours would have reached approximately $1 \mathrm{~cm}^{3}$ by the end of the study period had they been allowed to mature. Hence our heat triggered system would have resulted in a staggering 5 -fold reduction in tumour size.

Ideally, HNP-BNIPSpm without laser irradiation would not result in any tumour retardation for the clinical translation to occur. Hence, we postulate that surface modification using polymers to wrap the drugs onto the HNP surface until laser irradiation and subsequent release will improve their biocompatibility and reduce this observed toxicity. Work is underway in our laboratories in order to develop these second-generation systems. 


\section{Conclusion}

This study realises the potential of HNPs as vehicles for heat triggered drug delivery in pancreatic cancer therapy. In general, loading capacity of drugs was affected by a number of factors including initial drug feeding concentration, the extent of cationic charge (number of amino groups in the drug backbone), the presence of sulfur in the drug and the type of interaction between drug and HNPs (electrostatic or covalent). Our findings show that increasing the number of charges in the polyamine backbone leads to stronger electrostatic interaction with the gold surface of the HNP. BNIPSpm possessed favourable binding, which was reversible at $44^{\circ} \mathrm{C}$. This means the drug could be released by heat stimulation via irradiation at the SPR in vivo. The possibility of using these systems clinically is not limited to the novel bisnaphthalimide compounds. Indeed, any charged drug molecule which exhibits desirable binding and uncoupling characteristics could be delivered by this route. Additionally, active transport mechanisms for precision targeting could be exploited using specific ligands. Undoubtedly the surface chemistry is only limited by area and competition for binding sites. Exploitation of these systems for heat triggered drug delivery in combination with their imaging capability makes them suitable as theranostic agents.

The advantage of such systems is the ability to treat patients as soon as diagnosis is confirmed with more effective and targeting systems which results in reduction in the dosage required of these harsh cytotoxic agents in order to observe therapeutic effect, reduction in patient side effects and improved long-term outcome. In order for these systems to truly be classed as theranostics they must be completely biocompatible after drug conjugation until the point of laser initiated drug release. Hence, our lab are currently working on the next generation of these systems with careful attention to the surface chemistry and immunological response pushing this potential treatment further along the pipeline in order to create significant advancement in the treatment of pancreatic cancer which could be translated to other cancers.

\section{Acknowledgements}

The authors wish to acknowledge the EPSRC UK National Mass Spectrometry Facility at Swansea University. 


\section{Competing Interests}

The authors would like to state that they have no competing interests.

\section{References}

1. A. Jemal, A. Thomas, T. Murray and M. Thun, Cancer J. Clin., 2002, 52, 23-47.

2. PCUK, Taking on research to change the future: Five year research strategy, PCUK, 2017.

3. A. Sa Cunha, C. Rault, C. Laurent, X. Adhoute, V. Vendrely, G. Béllannée, R. Brunet, D. Collet and B. Masson, J. Am. Coll. Surg., 2005, 201, 359-365.

4. M. Malekigorji, ADM Curtis, C. Hoskins, J. Nanomed. Res. 2014, 1, 1.

5. S. Jain, D.G. Hirst and J.M. O’Sullivan, Br. J. Radiol., 2012, 85, 101-113.

6. A. Abruzzo, G. Zuccheri, F. Belluti, S. Provenzano, L. Verardi, F. Bigucci, T. Cerchiaria, B. Luppi and N. Calonghi, Colloids Surfaces B: Biointerfaces, 2016, 145, $362-372$.

7. A. Sarkar, S. Ghosh, S. Chowdhury, B. Pandey and P.C. Sil, Biochimica et Biophysica Acta, 2016, 1860, 2065 -2075.

8. R. Sun, S. Shen, Y.J. Zhang, C.F. Xu, Z.T. Cao, L.P. Wen and J. Wang, Biomaterials, 2016, 103, 44-55.

9. S. Duchi, S. Ramos-Ramero, B. Doxxa, M. Guerra-Rebollo, L. Cattini, M. Ballestri, P. Dambruoso, A. Guerrini, G. Sotgiu, G. Varchi, E. Lucarelli and J. Blanco, Nanomedicine: Nanotechnology, Biology, and Medicine, 2016, 12, 1885-1897.

10. V. Khare, W. Al-Shakarchi, P.M. Gupta, A.D.M. Curtis and C. Hoskins, RSC Adv., 2016, 6, 60126.

11. K. Greish, Methods Mol. Biol., 2010, 624, 25-37.

12. J.J. Byrne, T. Betancourt and L. Brannon-Peppas, Adv. Drug Deliver. Rev., 2008, 60, 1615-1626.

13. Y. Yang, A. Roy and S. Li, Front. Bioeng. Biotechnol. Conference Abstract: 10th World Biomaterials Congress, 2016.

14. F. Yuan, M. Leunig, S.K. Huang, D.A. Berk, D. Papahadjopoulos, and R.K. Jain, Cancer Res. 1994, 54, 3352-3356.

15. V.P. Chauhan, Z. Popovic, O. Chen, J. Cui, D. Fukumura, M.G. Bawendi, and R.K. Jain, Angew. Chem. Int. Ed. Engl., 2011, 50, 11417-11420.

16. C.L. Waite and C.M. Roth, Crit. Rev. Biomed. Eng., 2012, 40, 21-41. 
17. C. Hoskins, Y. Min, M. Gueorguieva, C. McDougall, A. Volovick, P. Prentice, Z. Wang, A. Melzer, A. Cuschieri and L. Wang, J. Nanobiotechnol., 2012, 10, 27.

18. C. Hoskins, L. Wang, W.P. Cheng, and A. Cuschieri, Nanoscale Res. Letts., 2012, 7 , 77.

19. M.S. Sajadi, F. Fathi and N. Farhadyar, IJCPS, 2014, 2, 760-764.

20. P. Štarha, D. Smola, J. Tucek and Z. Trávnícek, Int. J. Mol. Sci, 2015, 16, 2034-2051.

21. C.M. Barnett, M. Gueorguieva, M.R. Lees, D.J. McGarvey, R.J. Darton, and C. Hoskins, J. Nanopart. Res., 2012, 14, 1170.

22. C.M. Barnett, M. Gueorgieva, M.R. Lees, D.J. McGarvey and C. Hoskins, J. Nanopart. Res., 2013, 15, 1706.

23. A.J. Wagstaff, S.D. Brown, M.R. Holden, G.E. Craig, J.A. Plumb and R.E. Brown, Inorganica Chimica Acta., 2012, 393, 328-333.

24. H. Wennerström, Langmuir, 1991, 7, 3154-3159.

25. S.S. Banjeree, N. Aher, R. Patil and J. Khandare, J Drug Deliver, 2012, 103973, 1-17.

26. M.F. Brana, J.M. Castellano, C.M. Roldán, A. Santos, D. Vázquez and A. Jiménez, Cancer Chemotherapy and Pharmacology, 1980, 4, 61-66

27. M.F. Brana, J.M. Catellano, M. Morán, M.J.Pérez de Vega, X.D. Qian, C.A. Romerdahl and G. Keilhauer, Eur. J. Med. Chem., 1995, 30, 235-239.

28. M.F. Brana, J.M. Castellano, M. Morán, M.J. Pérez de Vega, C.R. Romerdagl, X.D. Qian, P. Bousquet, F. Emling, E. Schlick and G. Keilhauer, Anti-Cancer Drug Design, 1993, 8, 257-268.

29. M.F. Brana and A. Ramos, Current Medicinal Chemistry - Anti-Cancer Agents, 2001, 1, 237-255.

30. S.M. Sami, R.T. Dorr, D.S. Alberts, A.M. Sólyom and W.A. Remers, J. Med. Chem, 2000, 43, 3067-3073.

31. C. Hoskins, M. Ouaissi, S.C. Lima, W.P. Cheng, I. Loureirio, E. Mas, D. Lombardo, A. Cordeiro-Da-Silva, A. Ouaissi and P. Kong Thoo Lin, Pharm. Res., 2010, 27, 2694-2703.

32. P. Workman, E.O. Aboagye, F. Balkwill, A. Balain, G. Bruder, D.J. Chaplin, J.A. Double, J. Everitt, D.A.H. Farningham, M.J. Glennie, L.R. Kelland, V. Robinson, I.J. Stratford, G.M.Tozer, S. Watson, S.R. Wedge and S.A. Eccles, British Journal of Cancer, 2010, 102, 1555-1577.

33. P. Kong Thoo Lin and V.A. Pavlov, Bioorg Med Chem Lett, 2000, 10, 1609-1612. 
34. A.D.M. Curtis, M. Malekigorji, H. Holman, M. Skidmore and C. Hoskins, J. Nanomed. Nanotechnol., 2015, 6, 6.

35. A. Oluwasamni, M. Malekigorji, S. Jones, A.D.M. Curtis and C. Hoskins, RSC Adv., 2016, 6, 95044-95054.

36. Y. Guo, Z. Zhang, D-H. Kim, W. Li, J. Nicolai, D. Procissi, Y. Huan, G. Han, R.A. Omary and A.C. Larson, Int. J. Nanomedicine., 2013, 8, 3437-3446.

37. M. Aslam, et al., J. Mater. Chem, 2004, 14, 1795-1797.

38. A. Ulman, Chem. Rev, 1996, 96, 1533-1554.

39. D. Curry, A. Cameron, B. MacDonald, c. Nganou, H. Scheller, J. Marsh, S. Beale, M. Lu, Z. Shan, R. Kaliaperumal, H. Xu, m. Servos, c. Bennett, S. MacQuarrie, K.D. Oakes, M. Mkandawire and X. Zhang, Nanoscale, 2015, 7, 19611-9619.

40. N. Oh, J-H. Park, Int. J. Nanomedicine, 2014, 9, 51-63. 


\section{Figures}

Figure 1. A) Schematic representation of drug dissociation from hybrid nanoparticle surface after laser irradiation induced heating leading to reversal of electrostatic binding. B) Chemical structures of bisnaphthalimido based drugs of varied charge used for binding onto hybrid nanoparticle surface: 1. BNIPDi, 2. BNIPd, 3. BNIPSpm \& 4. BNIPds.

Figure 2. The synthetic strategy adopted to synthesise new bisnaphthalimides 2-4.

Figure 3. Physical properties of HNPs including A) ability to electrostatically bind charged drug molecules onto the surface, B) zeta potential measurement before and after drug conjugation and stability of formulations stored at C) $25{ }^{\circ} \mathrm{C}$ and D) $4{ }^{\circ} \mathrm{C}$ $(n=3, \pm S E)$.

Figure 4. In vitro drug release of BNIPSpm formulations A) in aqueous solution B) in physiological media at $37{ }^{\circ} \mathrm{C}$ (red) and $44{ }^{\circ} \mathrm{C}$ (blue) ( $n=3, \pm S E$ ). * denotes significance compared with all other temperatures tested $(p<0.01)$.

Figure 5. In vitro cellular evaluation on BxPC-3, Panc-1 and U937 cells. A) Cytotoxicity assays, B) cellular uptake study, C) thermally initiated cytotoxicity assay and D) AFM topography images using Scan Asyst in air mode on fixed 1) control cells, and cells incubated with 2) HNPs, 3) gemcitabine 4) BNIPSpm and 5) HNPBNIPSpm for $1 \mathrm{~h} .(n=3 \pm S E)$. * denotes significance compared to free drug, \# denotes significance compared to other temperatures tested $(p<0.01)$.

Figure 6. In vivo evaluation on BxPC-3 xenograft models in $\mathrm{Nu} / \mathrm{Nu}$ 4-6 week old female mice dosed once a week at $3 \mathrm{mgKg}^{-1}$ over 4 weeks. A) Comparison of tumours after excision: 1) control, 2) control with laser irradiation, 3) HNP, 4) HNP with laser irradiation, 5) BNIPSpm, 6) BNIPSpm with laser irradiation, 7) HNP-BNIPSpm, 8) HNP-BNIPSpm with laser irradiation. Solid line: no laser irradiation, dashed line: laser irradiation. Where laser irradiation was required this was carried out $24 \mathrm{~h}$ after dosing under anaesthetic. The tumour was irradiated at $1064 \mathrm{~nm}$ as for $20 \mathrm{sec}$ using a ML-LASER-YB5 Q-switched Nd:YAG Laser Treatment System. Pulse width: $10 \mathrm{~ns}$, pulse repetition frequency: $6 \mathrm{~Hz}$, laser spot diameter: $3 \mathrm{~mm}$, cooling system: water cooled with airflow cooling. The beam was collimated through concave lenses to a $1 \mathrm{~mm}$ diameter. B) Comparison of tumour weight after excision and $C)$ comparison of tumour volume over study duration, $n=5 \pm S E$ ). $\infty$ Study stopped before completion due to tumour volume approaching maximum humane volume $\left(0.9 \mathrm{~cm}^{3}\right)$. * denotes significance compared to controls, \# denotes significance compared to free drug, $\measuredangle$ denotes significance compared to HNP-BNIPSpm without laser irradiation. 
Figures.

A

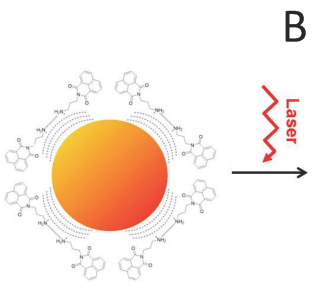

B

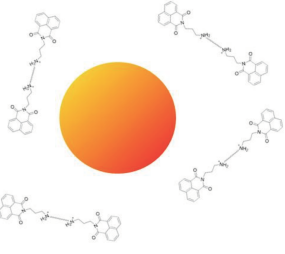

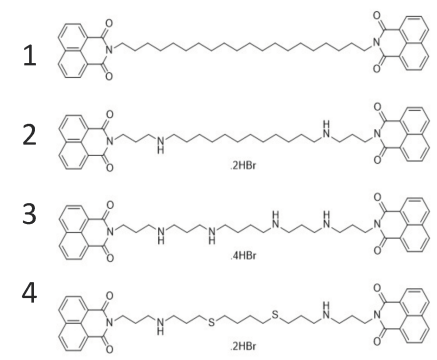

2 -

3 She zitar

Figure 1. 

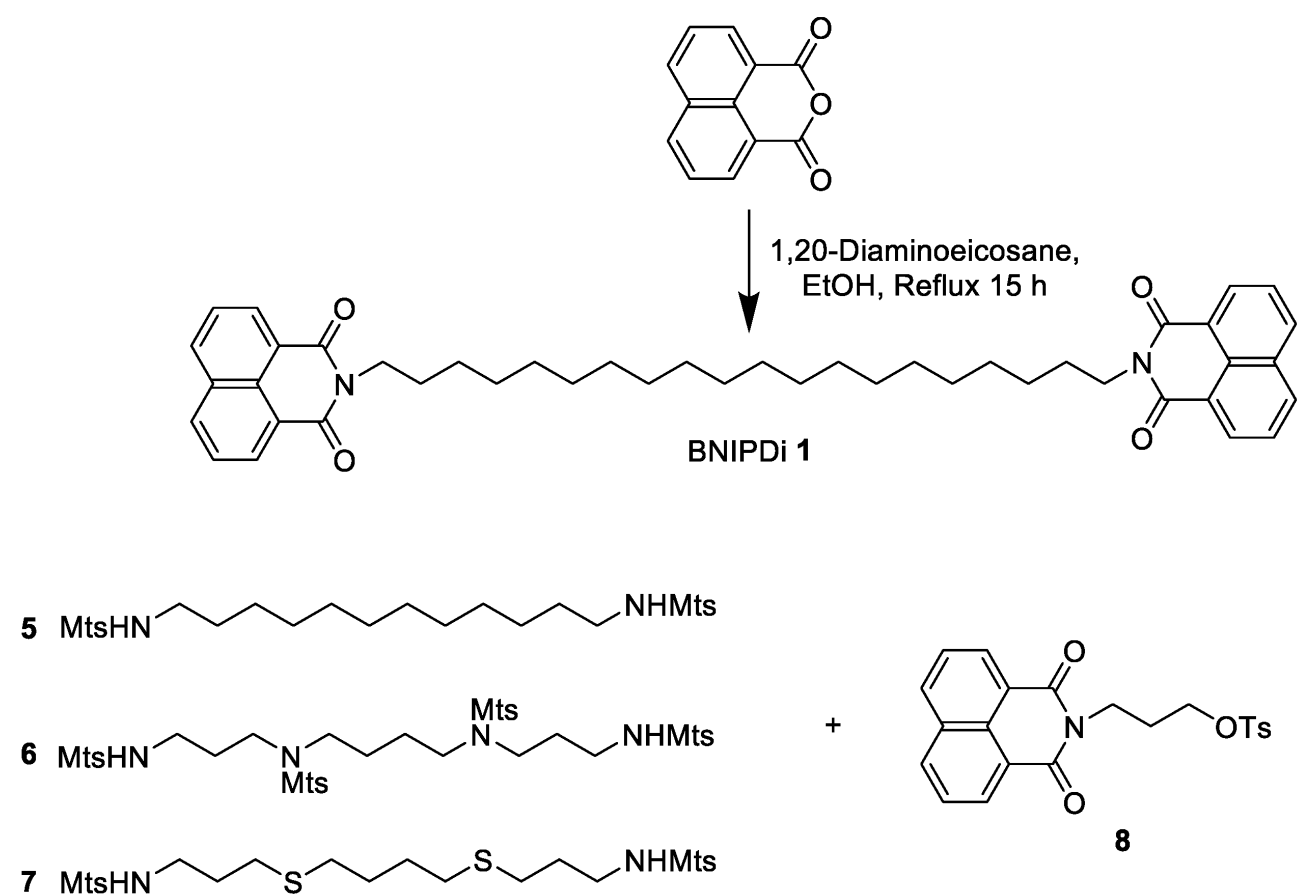

1. $\mathrm{Cs}_{2} \mathrm{CO}_{3}, \mathrm{DMF}, 80^{\circ} \mathrm{C}, 12 \mathrm{~h}$

2. $\mathrm{HBr} 33 \%$ solution in $\mathrm{AcOH}, \mathrm{CH}_{2} \mathrm{Cl}_{2}, 25^{\circ} \mathrm{C}, 18 \mathrm{~h}$<smiles>CCCCCCCCCCCCCCCCNCCCN1C(=O)c2cccc3cccc(c23)C1=O</smiles><smiles>CCCCCCCCCCNCCCCNCCCNCCCNCCCN1C(=O)c2cccc3cccc(c23)C1=O</smiles><smiles>CCCCCCCCSCCCCSCCCNCCCN1C(=O)c2cccc3cccc(c23)C1=O</smiles>

Figure 2. 
A

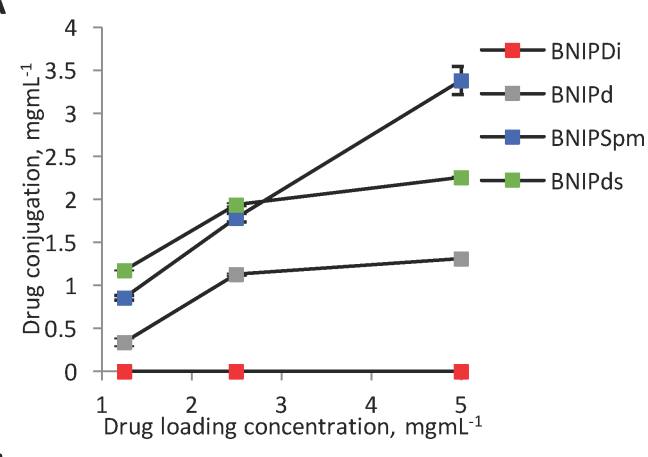

C

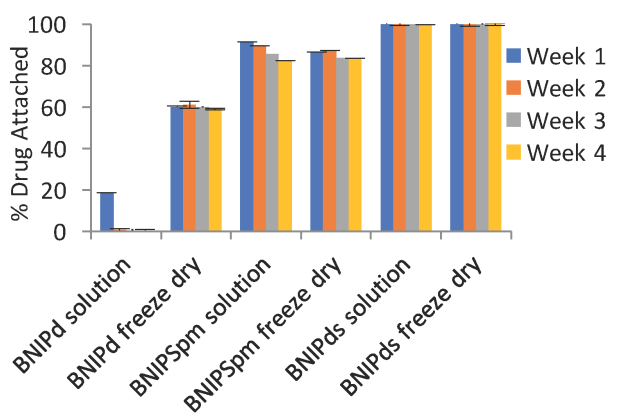

B

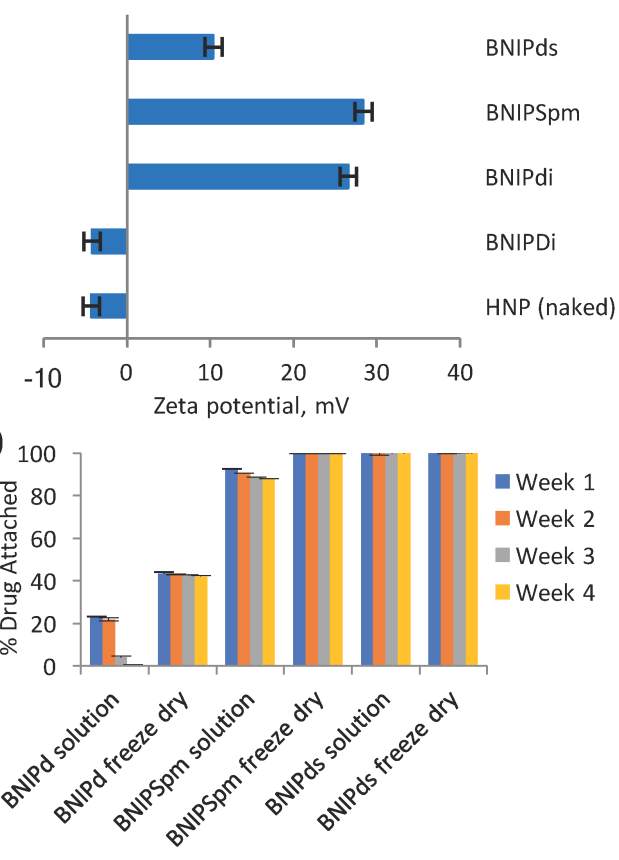

Figure 3.
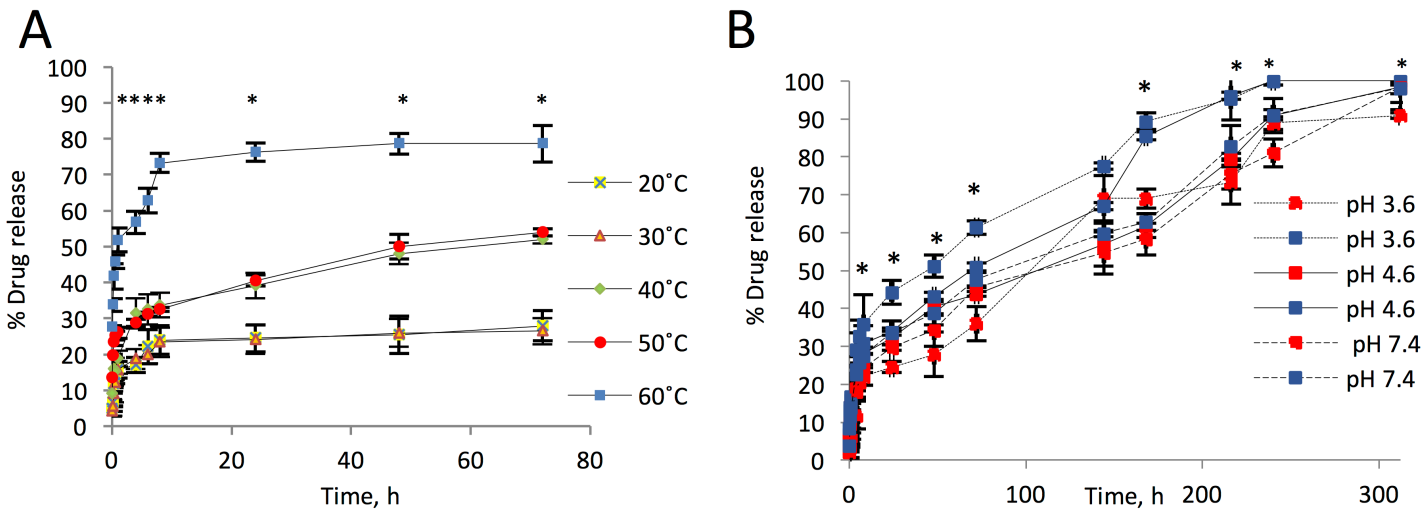

Figure 4. 


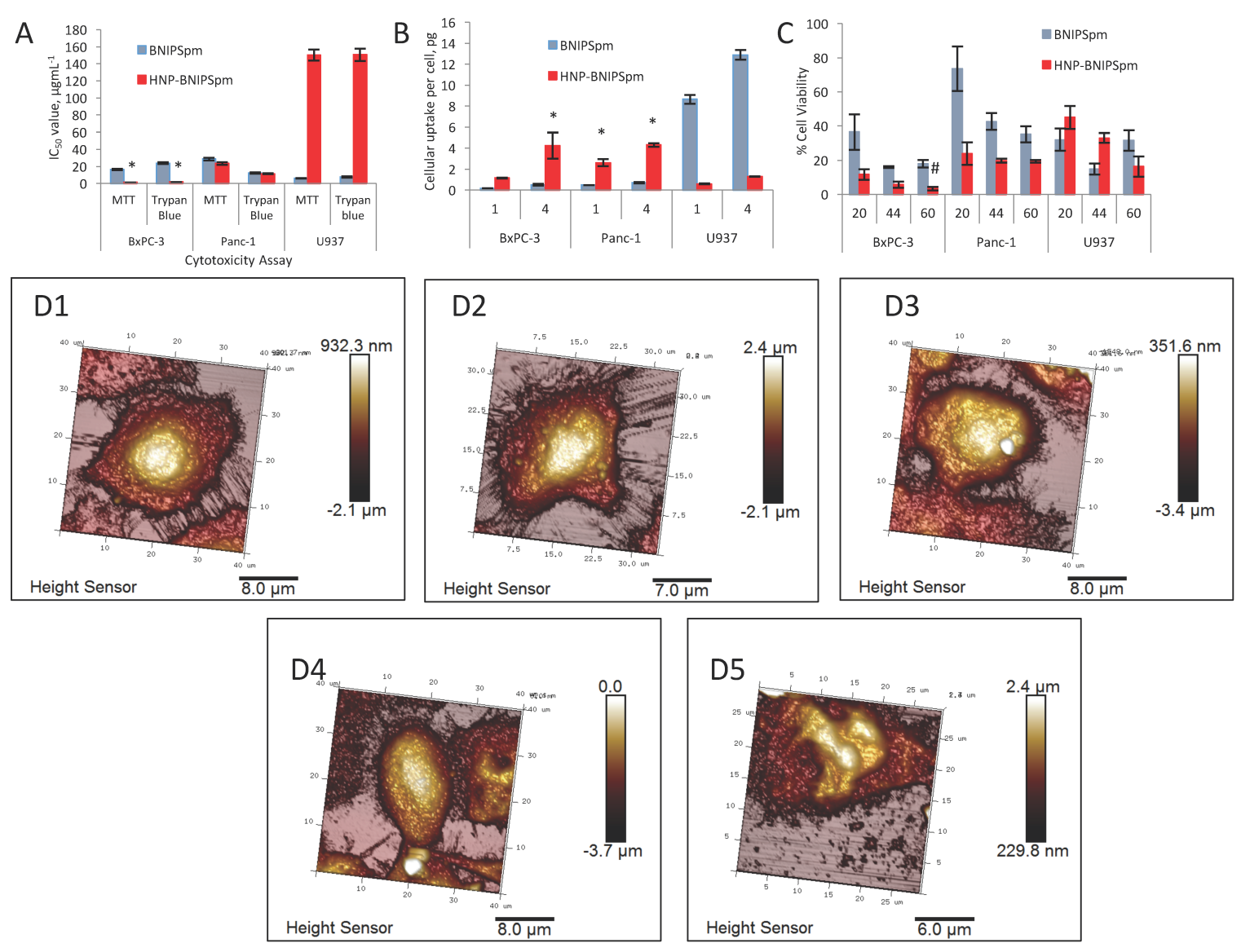

\section{Figure 5.}
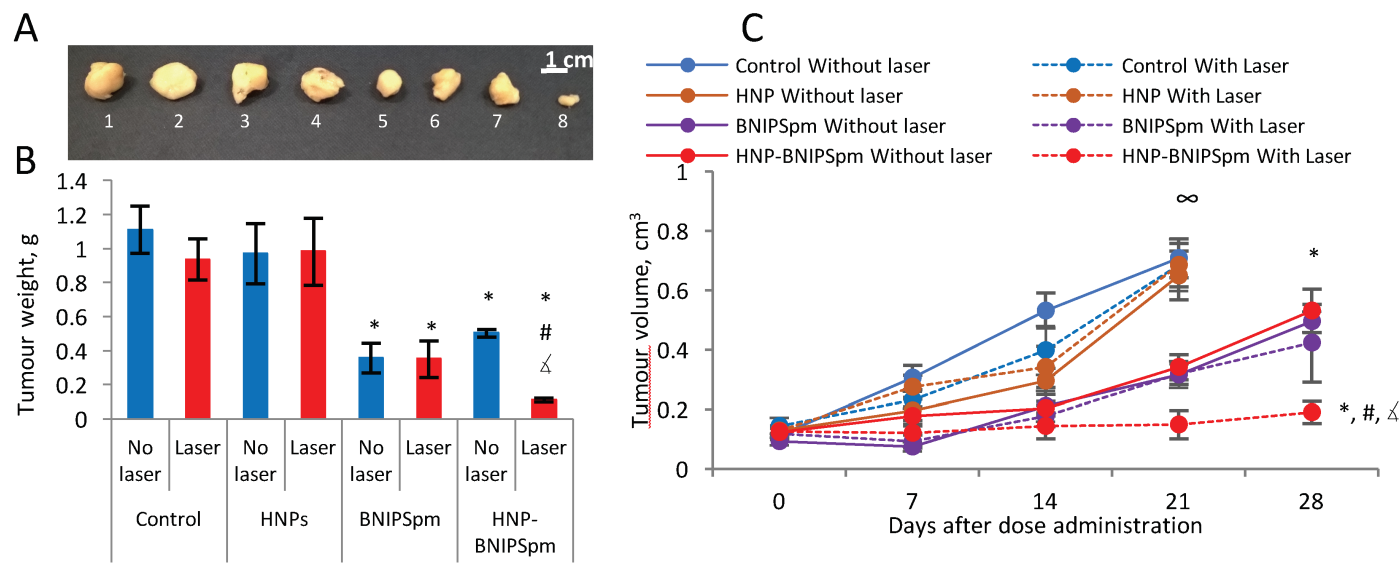

Figure 6. 